\title{
The use of a UV-C disinfection robot in the routine cleaning process: a field study in an Academic hospital
}

Füszl Astrid, Zatorska Beata, Van den Nest Miriam, Ebner Julia, Presterl Elisabeth * (10 and Diab-Elschahawi Magda

\begin{abstract}
Background: Environmental surface decontamination is a crucial tool to prevent the spread of infections in hospitals. However, manual cleaning and disinfection may be insufficient to eliminate pathogens from contaminated surfaces. Ultraviolet-C (UV-C) irradiation deploying autonomous disinfection devices, i.e. robots, are increasingly advertised to complement standard decontamination procedures with concurrent reduction of time and workload. Although the principle of UV-C based disinfection is proven, little is known about the operational details of UV-C disinfection delivered by robots. To explore the impact of a UV-C disinfection robot in the clinical setting, we investigated its usability and the effectiveness as an add-on to standard environmental cleaning and disinfection. Additionally, its effect on Candida auris, a yeast pathogen resistant to antifungals and disinfectants, was studied.

Methods: After setting the parameters "surface distance" and "exposure time" for each area as given by the manufacturer, the robot moved autonomously and emitted UV-C irradiation in the waiting areas of two hospital outpatient clinics after routine cleaning and/or disinfection. To quantify the efficacy of the robotic UV-C disinfection, we obtained cultures from defined sampling sites in these areas at baseline, after manual cleaning/disinfection and after the use of the robot. Four different $C$. auris strains at two concentrations and either in a lag or in a stationary growth phase were placed in these areas and exposed to UV-C disinfection as well.

Results: The UV-C irradiation significantly reduced the microbial growth on the surfaces after manual cleaning and disinfection. C. auris growth in the lag phase was inhibited by the UV-C irradiation but not in the presence of the rim shadows. The effects on C. auris in the stationary phase were differential, but overall C. auris strains were not effectively killed by the standard UV-C disinfection cycle. Regarding usability, the robot's interface was not intuitive, requiring advanced technical knowledge or intensive training prior to its use. Additionally, the robot required interventions by the technical operator during the disinfection process, e.g. stopping due to unforeseen minor dislocation of items during the clinical service or due to moving individuals, making it a delicate high-tech device but not yet ready for the autonomous use in the clinical routine.
\end{abstract}

Conclusions: Presently, the UV-C robot tested in this study is not ready to be integrated in the environmental cleaning and disinfection procedures in our hospital. The single standard disinfection UV-C irradiation cycle is not sufficient to inactivate pathogens with augmented environmental resilience, e.g. C. auris, particularly when microbial loads are high.

Keywords: Healthcare-associated infections, Infection control, Ultraviolet-C, UV-C robot, Candida auris

*Correspondence: elisabeth.presterl@meduniwien.ac.at

Department of Infection Control and Hospital Epidemiology, Medical

University Vienna, Währinger Gürtel 18-20, 1090 Vienna, Austria original author(s) and the source, provide a link to the Creative Commons licence, and indicate if changes were made. The images or other third party material in this article are included in the article's Creative Commons licence, unless indicated otherwise in a credit line to the material. If material is not included in the article's Creative Commons licence and your intended use is not permitted by statutory regulation or exceeds the permitted use, you will need to obtain permission directly from the copyright holder. To view a copy of this licence, visit http://creativecommons.org/licenses/by/4.0/. The Creative Commons Public Domain Dedication waiver (http://creativeco mmons.org/publicdomain/zero/1.0/) applies to the data made available in this article, unless otherwise stated in a credit line to the data. 


\section{Introduction}

Healthcare-associated infections (HAIs) are a major complication of medical treatment and care, necessitating a prolonged hospital stay and causing morbidity associated with increased costs and last but not least increased mortality [1]. Up to 7\% of the patients in developed and $10 \%$ of the patients in developing countries are at risk to acquire at least one HAI, most of which may be prevented through infection prevention and control (IPC) measures [2].

Pathogens, e.g. methicillin-resistant Staphylococcus aureus (MRSA), vancomycin-resistant Enterococcus (VRE), Clostridium difficile, Norovirus and fungi are viable on surfaces for prolonged periods [3-6]. As a result, environmental contamination leads to an increased risk of HAIs [3, 7]. To prevent HAIs and the spread of pathogens via contaminated surfaces, hospital rooms have to be cleaned and disinfected at regular intervals by trained personnel. For decontamination in hospitals, cleaning agents and disinfectants approved by technical expert committees must be used. However, manual cleaning and disinfection is time and personnel consuming anddue to lack of time and training-sometimes not sufficient. Erratic cleaning and disinfection processes, wrong choice of the appropriate formulation of cleansers or disinfectants and non-adherence to the required contact time of disinfectants may impair the efficacy of standard approaches. Studies have shown that more than $50 \%$ of surfaces may go untouched by manual cleaning $[3,8,9]$. Secondly, in times of crisis, the supply of disinfectants may be disrupted, as has been demonstrated in the current COVID-19 pandemic [10].

Because of the shortcomings of routine environmental decontamination as mentioned above, autonomous touchless surface disinfection technologies have evolved. By disrupting the structure of DNA or RNA of microorganisms, UV-C irradiation at a wavelength of $254 \mathrm{~nm}$ is most effective in killing bacteria, viruses, fungi, and even spores (in falling order of effectiveness) [11].

Previous studies indicate that disinfection technologies using UV-C irradiation are an enhancement to standard cleaning and disinfection, reducing the environmental microbial burden and potentially mitigating the risk of acquiring a HAI [12-18]. This has been demonstrated for different pathogens such as MRSA, Clostridium difficile and VRE $[13,18]$ and in different clinical settings such as ambulances [19], inpatient rooms [16, 20] and operating theaters [15].

The efficacy of UV-C irradiation to inactivate microorganisms depends on a number of factors including varying resistance levels of different microorganisms to UV-C light, the initial inoculum and the UV-C dose received, which is a result of distance, duration of exposure and the presence of shadows [21]. Organic soils, furniture, draperies or other healthcare equipment etc. are the most common cause of shadows. Shadows drastically reduce the efficacy of UV-C irradiation. To remove soils, surfaces must be cleaned manually before applying UV-C irradiation. UV-C efficacy also declines with increasing distance of the UV-C source to the surfaces.

Candida auris is an emerging, multidrug-resistant yeast pathogen first described in 2009 as the cause of multiple nosocomial outbreaks worldwide, leading to severe infections and high mortality rates [22]. C. auris poses a particular challenge for IPC in hospitals because it can stay viable on surfaces for prolonged periods and is resistant to several commonly used disinfectants [25]. Consequently, the hospital environment is considered an important reservoir for transmission [22-25]. Further, compared to other pathogens, C. auris is resistant to $\mathrm{UV}-\mathrm{C}$ light and needs extended exposure to UV-C irradiation to induce growth inhibition $[26,27]$.

UV-C disinfection robots have been increasingly employed in different settings such as hospitals, airports and shopping malls as a result of the COVID-19 pandemic [28]. However, little information is available on their efficacy and usability in the routine cleaning and disinfection process in hospital settings. To shed some further light on operational aspects, we aimed to test a new UV-C robot in real life. To evaluate the antimicrobial efficacy of a standard UV-C disinfection cycle, we investigated its effect on the microbial burden on clinical surfaces when applied after standard terminal cleaning and disinfection (STC\&D) in the waiting areas of two outpatient clinics. As a surrogate for resilient microorganisms, four different $C$. auris strains in varying densities $\left(10^{3}\right.$ and $10^{6} \mathrm{CFUs} / \mathrm{ml}$ ) and different growth characteristics (lag vs stationary growth phase) were placed within these areas and exposed to UV-C irradiation as well.

\section{Materials and methods \\ UV-C light emitting disinfection device}

We studied the self-driving Ultra Violet Disinfection Robot $^{\circledR}$ (UVD-R) by Clean Room Solutions because it was the most advanced UV-C irradiation device available for autonomous use (Fig. 1).

This robot moves autonomously in a pre-defined area after being programmed for the parameters exposure time and distance of surfaces. It consists of eight lamps that are located on top of a platform. During a disinfection cycle, they emit UV-C irradiation at a wavelength of $254 \mathrm{~nm}$, enabling a 360 degree coverage. During the disinfection process, the UV-C light emitting robot moves at $10 \mathrm{~cm}$ per second, providing a dose of $2.7 \mathrm{~mJ} / \mathrm{cm}^{2}$ per second for directly exposed surfaces in $1 \mathrm{~m}$ distance and achieving a coverage of areas at a distance of several 


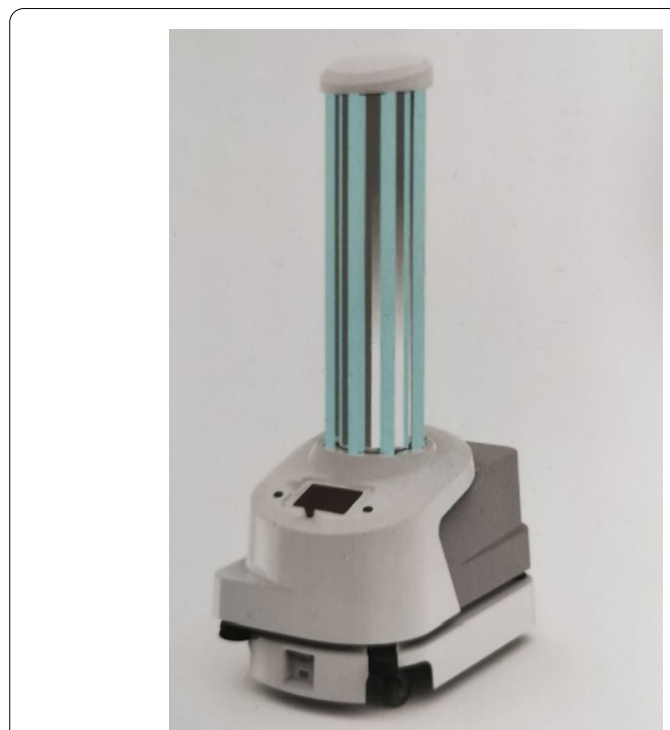

Fig. 1 UVD Robot ${ }^{\circledR}$ (Clean Room Solutions)

meters (according to manufacturer's specifications). However, it is worth bearing in mind that the UV-C light intensity over distance is governed by the inverse square law, resulting in significantly smaller doses for areas further away from the device. To enable autonomous moving, the robot must be pre-programmed using a detailed map of the position of furniture and other obstacles in the area to be treated with UV-C irradiation. Once every parameter is set, furniture and all other objects must remain in exactly the same place to enable an autonomous functioning. Due to the high-intensity UV-C irradiation, the UV-C robot may only be used in rooms devoid of people. Unintentional exposure leads to cutaneous erythema and photokeratitis. For safety, this UV-C robot automatically shuts off when its motion sensor detects any moving individuals during the disinfection process.

\section{Setting}

Between July 23rd and August 2nd 2020, the study was performed in the waiting areas of two outpatient clinics (ear, nose and throat medicine and oncology waiting areas with a size of $137 \mathrm{~m}^{2}$ each) of Vienna General Hospital (VGH), a 1728 bed tertiary hospital in the capital of Austria. During the study period, 347 patients were treated in the ENT (23/07-26/07) and 400 patients in the oncology outpatient department (29/07-02/08).

In one of the outpatient areas, manual cleaning/disinfection was carried out by in-house cleaning personnel while the other outpatient clinic was served by a cleaning service providing company. Cleaning and disinfection followed the standard operating procedures (SOP) of VGH: Floors in the outpatient waiting areas are manually cleaned once a day while chairs and tables are disinfected once a day using either alcohol-based products or products based on active oxygen (Descogen ${ }^{\circledR}$ Liquid).

Prior to the start of the study, a member of the UVD Robot $^{\circledR}$ installation team pre-programmed area maps with the exact position of furniture and other items to enable autonomous disinfection cycles. For the pre-programming, the team inspected the two outpatient clinics to map the robot's route and identify critical areas that required longer UV-C light exposure. The robot was preprogrammed to stop at various predefined positions for 3 min to achieve optimal UV-C exposure of all relevant surfaces (Figs. 2, 3).

The device was used after the room had been manually cleaned and disinfected according to SOP. The procedure was then initiated remotely once all doors had been

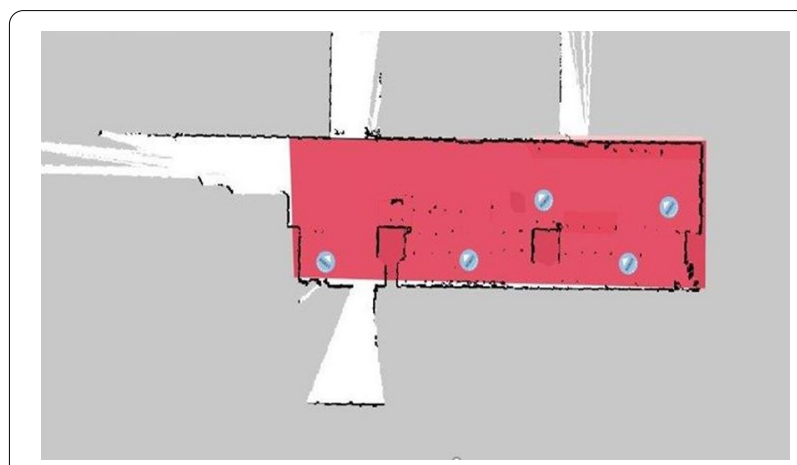

Fig. 2 Area map pre-programmed into the UV-C robot in the ENT outpatient clinic. The blue dots indicate where the robot had to stop for 3 min during the disinfection cycle. The red color indicates which area was covered by the mapping procedure and exposed to UV-C light

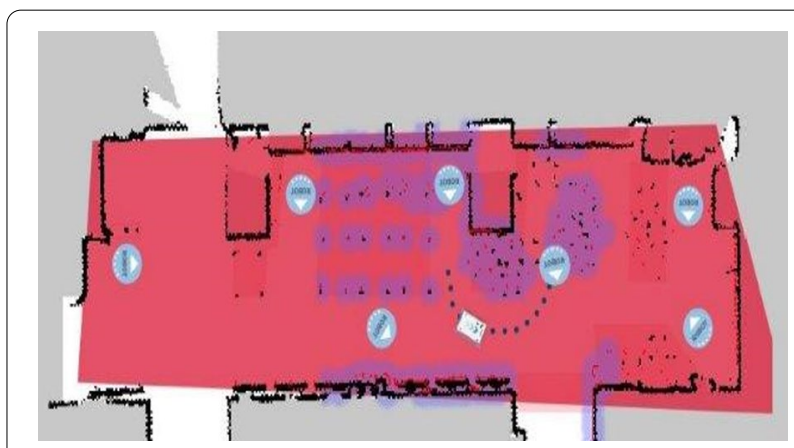

Fig. 3 Area map pre-programmed into the UV-C robot in the oncology outpatient clinic. The blue dots indicate where the robot had to stop for 3 min during the disinfection cycle. The red color indicates which area was covered by the mapping procedure and exposed to UV-C light. The violet color indicates "light detection and ranging" (Lidar), which is a way for the robot to see an obstacle and avoid that area 
closed. Each disinfection cycle was completed within 20-25 min per outpatient setting.

\section{Sampling procedure}

To evaluate the robot's effect on residual contamination, samples were collected from different surface sites before and after routine cleaning and/or disinfection, and after the use of the UV-C robot. Surface sites selected for sampling included high-touch surfaces and remote sites supposedly to be out of reach for easy cleaning and those that appeared unlikely to achieve full exposure to UV-C irradiation.

In the ENT waiting area, six sites were sampled (wall, armrests of two different chairs, back of a chair, wooden play element for children, window countertop) (see Additional file 1: Table S1).

In the oncology waiting area, sampling was performed from eight different sites (patient registration area, table surface next to patient registration, armrests of two different chairs, window countertop, push button of a vending machine, leaflet dispenser) (see Additional file 1: Table S2).

To monitor the amount of exposure to UV-C irradiation, disposable indicators were placed on all surfaces used for sampling before initiating the UV-C cycle. The indicators changed color depending on the achieved dose, corresponding to doses ranging from $25 \mathrm{~mJ} / \mathrm{cm}^{2}$ in shadowed areas to $100 \mathrm{~mJ} / \mathrm{cm}^{2}$ at the most highly exposed sites (Fig. 4).

The achieved UV-C doses corresponding to each sampling site are presented in the supplemental material (see Additional file 1: Tables S1-S2).

\section{Microbiological methods}

\section{Determination of the microbial burden on hospital surfaces}

We collected environmental contact cultures from each sampling site using Tryptic Soy Agar (TSA) plates with a diameter of $5.5 \mathrm{~cm}$ (VWR International, Vienna, Austria). Samples were collected on 9 days by the same lab technician following a predefined standardized sampling scheme:

During the study period, sampling was performed three times per study day:

(a) before routine cleaning and/or disinfection,

(b) after routine cleaning and/or disinfection, and

(c) after the use of the UV-C robot.

After sampling, TSA plates were incubated at $37{ }^{\circ} \mathrm{C}$ for $48 \mathrm{~h}$. Following incubation, the number of colony forming units (CFUs) on each plate was counted. Subsequently, the colonies were identified using the MALDITOF mass spectrometry method (Bruker, USA).

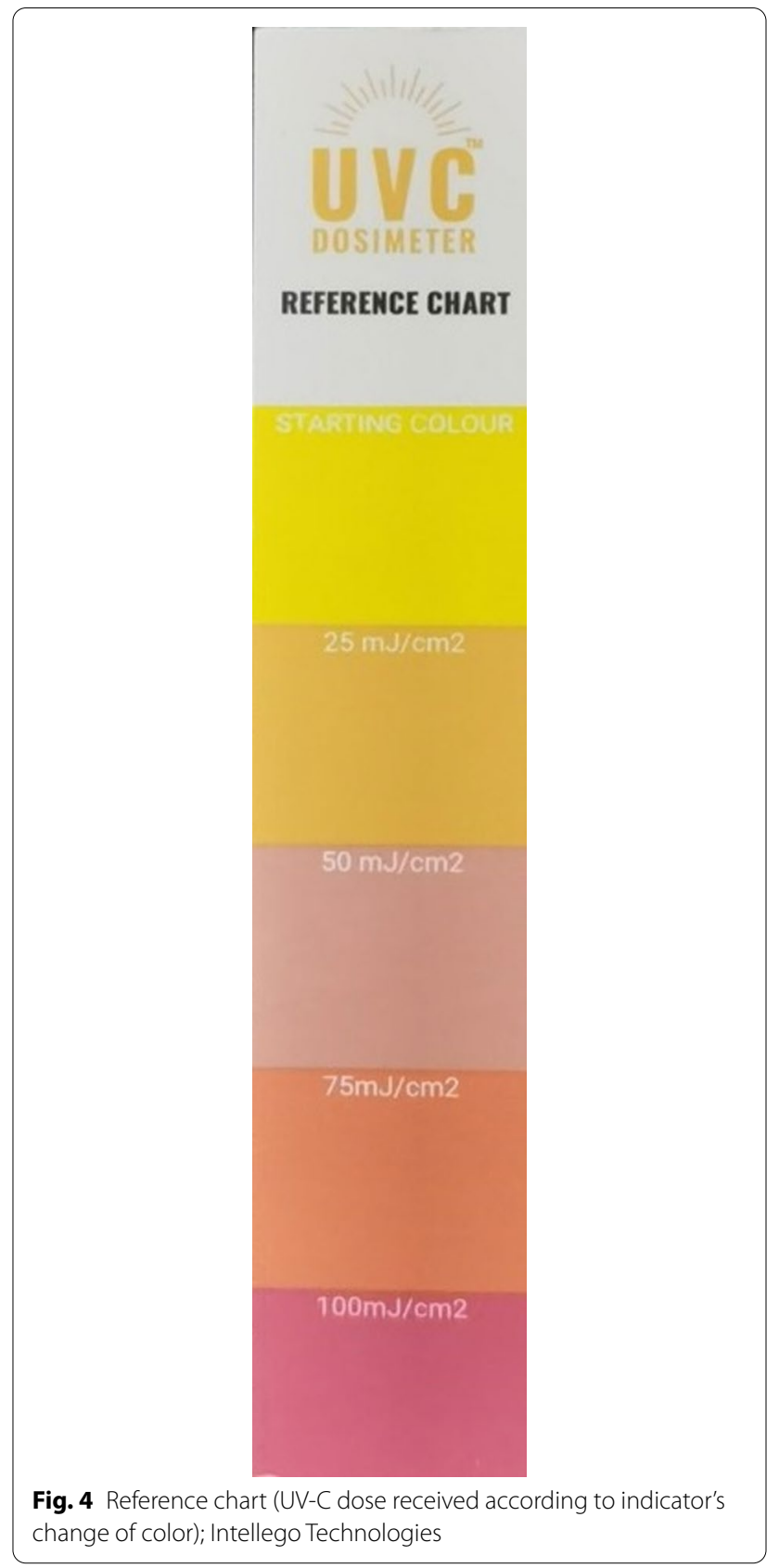

Surfaces were subdivided into three categories according to their level of contamination used routinely for environmental samples at our institution:

(1) surfaces with a low microbial burden, defined as $0-3$ CFUs $/ 24 \mathrm{~cm}^{2}$

(2) surfaces with an average microbial burden, defined as $4-50 \mathrm{CFUs} / 24 \mathrm{~cm}^{2}$

(3) surfaces with a high microbial burden, defined as $>50 \mathrm{CFUs} / 24 \mathrm{~cm}^{2}$. 


\section{Preparation of Candida auris strains}

We investigated whether the type of $C$. auris strain, seeding density and incubation prior to UV-C light exposure affected UV-C efficacy.

To study potential differences in sensitivity to UV-C irradiation, four $C$. auris strains were evaluated: $C$. auris NCPF 8971, C. auris NCPF 8977, C. auris NCPF 8984 and C. auris DSM 21092.

Plates containing Sabouraud Dextrose Agar (SDA) (Becton Dickinson, Franklin Lakes, USA) were inoculated with $100 \mu \mathrm{l}$ of $C$. auris suspension at two different concentrations, $10^{3} \mathrm{CFUs} / \mathrm{ml}$ and $10^{6} \mathrm{CFUs} / \mathrm{ml}$ respectively.

Each strain of $C$. auris suspension containing $10^{3}$ CFUs/ml was spread on three SDA plates and incubated for $24 \mathrm{~h}$ at $30{ }^{\circ} \mathrm{C}$. For the field experiment, rimless TSA plates were pressed on these SDA plates, mimicking surface contamination by hands and fomites as demonstrated by Adams et al. [29]. Overall, 12 TSA plates were used per study day.

Additionally, each strain of $C$. auris suspension containing $10^{6} \mathrm{CFUs} / \mathrm{ml}$ was spread on one SDA plate without further incubation, yielding four plates with $C$. auris in a lag phase per study day. Then, $C$. auris exposed TSA plates $\left(10^{3} \mathrm{CFUs} / \mathrm{ml}\right.$, incubated overnight) as well as inoculated SDA plates $\left(10^{6} \mathrm{CFUs} / \mathrm{ml}\right.$ without further incubation) were placed on two tables in the waiting area of the oncology outpatient clinic during the standard disinfection cycle. Indicators were placed alongside that measured the UV-C dose received. This experiment was performed in triplicate.

Following UV-C exposure, all plates were incubated at $30{ }^{\circ} \mathrm{C}$ for 7 days. Then, $C$. auris growth was compared to unexposed controls.

\section{Statistical analysis}

Standard descriptive analysis was done to summarize the microbiological findings. Differences between the number of CFUs after standard terminal cleaning and disinfection compared to the combined use of STC\&D and UV-C irradiation were analyzed using the Wilcoxon matched-pairs signed rank test. Statistical analysis was performed at a two-sided significance level of 0.05 , using SPSS (Version 26.0, IBM).

In an exploratory data analysis, the plates of UV-C exposed C. auris strains with a concentration of $10^{6}$ CFUs/ml were visually compared to unexposed controls. Results of $C$. auris with an initial concentration of $10^{3}$ $\mathrm{CFUs} / \mathrm{ml}$ were quantified as the number of CFUs, comparing exposed and unexposed plates.

\section{Results}

\section{Effects on the environmental microbial burden}

During the study period, we collected 192 samples (72 in the ENT and 120 in the oncology outpatient areas, respectively) from 14 sites (64 samples prior to any cleaning and disinfection, 64 after manual cleaning and disinfection and 64 after the use of the UV-C robot). Prior to manual cleaning, the surfaces most heavily contaminated were the armrests of chairs, followed by the window countertops. The least contaminated sites were the walls, the leaflet dispenser and the backs of patient chairs. The leaflet dispenser, however, was empty during the study period according to an in-house IPC order to avoid potential cross-transmission via contaminated leaflets during the COVID-19 pandemic. In general, contamination levels prior to any cleaning and disinfection were higher in the oncology outpatient area than in the ENT outpatient area.

In Table 1, the level of contamination according to the time of sampling is summarized for each outpatient waiting area. UV-C indicators showed that some of the sites received a suboptimal UV-C dose. Nonetheless, the additional use of UV-C irradiation achieved a further reduction of CFUs compared to standard cleaning and/ or disinfection, resulting in decontamination of $96.9 \%$ $(62 / 64)$ of the surfaces compared to decontamination of $50.0 \%(32 / 64)$ of the surfaces after manual cleaning and disinfection alone.

With regard to the microbial burden, the additional use of the UV-C robot significantly decreased the median number of CFUs in both outpatient areas compared to manual cleaning and disinfection alone $(p=0.008$ and

Table 1 Proportion of contact cultures with low, acceptable and high microbial burden before routine cleaning and/or disinfection, after routine cleaning and/or disinfection and after the use of the UV-C robot

\begin{tabular}{|c|c|c|c|c|c|c|}
\hline & \multicolumn{3}{|c|}{ ENT outpatient area } & \multicolumn{3}{|c|}{ Oncology outpatient area } \\
\hline & Low & Average & High & Low & Average & High \\
\hline Before C\&D & $45.8 \%(11 / 24)$ & $37.5 \%(9 / 24)$ & $16.7 \%(4 / 24)$ & $22.5 \%(9 / 40)$ & $60.0 \%(24 / 40)$ & $17.5 \%(7 / 40)$ \\
\hline After C\&D & $79.2 \%(19 / 24)$ & $20.8 \%(5 / 24)$ & $0 \%(0 / 24)$ & $32.5 \%(13 / 40)$ & $62.5 \%(25 / 40)$ & $5.0 \%(2 / 40)$ \\
\hline After C\&D+ UV-C & $100 \%(24 / 24)$ & $0 \%(0 / 24)$ & $0 \%(0 / 24)$ & $95.0 \%(38 / 40)$ & $5.0 \%(2 / 40)$ & $0 \%(0 / 40)$ \\
\hline
\end{tabular}

$C \& D$ cleaning and disinfection, UV-C ultraviolet C; low $=0-3 \mathrm{CFUs} / 24 \mathrm{~cm}^{2}$, average $=4-50 \mathrm{CFUs} / 24 \mathrm{~cm}^{2}$, high $>50 \mathrm{CFUs} / 24 \mathrm{~cm}{ }^{2}$ 
$p<0.001$, for the ENT and for the oncology outpatient areas, respectively) (Table 2 ).

\section{Qualitative description of the environmental microbiome}

Most bacterial isolates were classified as physiological skin flora $(222 / 297 ; 74.7 \%)$. Next, $13.1 \%$ of bacteria were classified as environmental microorganisms (39/297), $6.4 \%$ of bacteria were classified as oropharyngeal flora $(19 / 297)$ and $5.7 \%$ of bacteria as potential pathogens $(17 / 297)$.

Typical pathogens were Staphylococcus saprophyticus $(\mathrm{n}=5)$ and Staphylococcus lugdunensis $(\mathrm{n}=1)$, Acintetobacter baumanii $(\mathrm{n}=2)$, Aerococcus viridans $(\mathrm{n}=1)$, Streptococcus pneumonia $(\mathrm{n}=1)$, Staphylococcus aureus $(\mathrm{n}=1)$ and Enterococcus casseliflavus $(\mathrm{n}=1)$. The armrests of chairs were the sites most frequently contaminated with pathogens. All identified microorganisms in both waiting areas, the median CFUs and the achieved UV-C doses, reported separately for each sampling site, time of sampling and outpatient waiting area, are given in the supplemental material (Additional file 1: Tables S1-S4).

\section{Effects on Candida auris}

UV-C irradiation emitted by the robot reduced the growth of all four $C$. auris strains spread at a concentration of $10^{6} \mathrm{CFUs} / \mathrm{ml}$ on SDA plates, mimicking the microbial lag phase. However, as shown in Fig. 5, growth of $C$. auris was observed on one fourth of the plate. According to the indicators placed alongside, the measured UV-C dose was $100 \mathrm{~mJ} / \mathrm{cm}^{2}$ (indicating maximum exposure) except for the area right next to the rim of the plate, demonstrating the shadow effect of the rim.

The effect of the UV-C robot on stationary C. auris cells at an initial concentration of $10^{3} \mathrm{CFUs} / \mathrm{ml}$ was variable and depended on the C. auris strain tested (Table 3). The $C$. auris NCPF 8984 strain was the most sensitive of the tested strains. It also showed the most consistent results regarding growth reduction after UV-C exposure compared to control plates. C. auris NCPF 8971

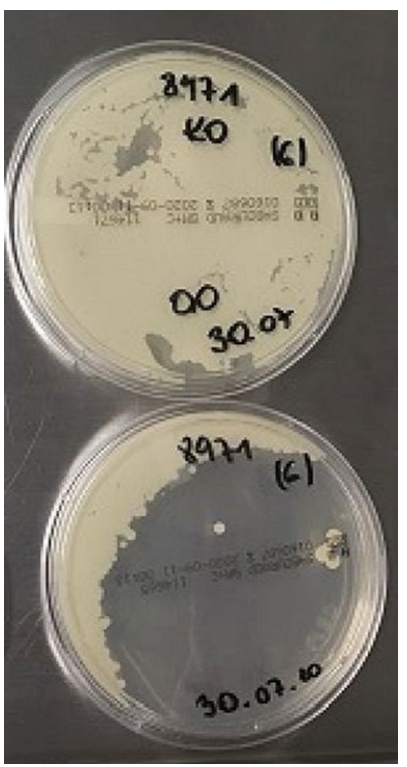

Fig. 5 C. auris $\left(10^{6} \mathrm{CFUs} / \mathrm{ml}\right)$ on Sabouraud plates without (above) and with (below) exposure to UV-C irradiation following incubation

consistently showed growth greater than 50 CFUs on each TSA contact plate after UV-C exposure. In terms of the UV-C dose received, the indicators indicated high exposure $\left(75-100 \mathrm{~mJ} / \mathrm{cm}^{2}\right)$ for all C. auris plates (Table 3, Fig. 6).

\section{Use of a UV-C robot for the routine cleaning and/ or disinfection process}

The UV-C robot required many attempts until it could carry out the UV-C disinfection process independently. Interventions by the operator were necessary due to initial programming imprecisions, furniture that had accidentally been moved during routine clinical operations, detection of movement during an ongoing disinfection cycle or loss of internet connection. Although the area was cordoned off during the disinfection cycles and appropriate warning signs were posted on the access doors, we found it difficult to

Table 2 Reductions in Colony-Forming Units after routine cleaning and/or disinfection compared to baseline and after routine cleaning and/or disinfection + UV-C irradiation compared to routine cleaning and/or disinfection alone

\begin{tabular}{|c|c|c|c|c|c|c|c|c|c|c|}
\hline & \multicolumn{5}{|c|}{ ENT outpatient area } & \multicolumn{5}{|c|}{ Oncology outpatient area } \\
\hline & $\begin{array}{l}\text { No. of } \\
\text { samples }\end{array}$ & Median CFU (IQR) & Min & Max & $p$ value & $\begin{array}{l}\text { No. of } \\
\text { samples }\end{array}$ & Median CFU (IQR) & Min & Max & $p$ value \\
\hline Before $C \& D$ & 24 & $8.5(8.5-28.3)$ & 0 & 207 & 0.003 & 40 & $22.0(4.3-36.0)$ & 0 & 200 & \\
\hline After C\&D & 24 & $0(0-2.8)$ & 0 & 18 & & 40 & $6.5(2.3-20.5)$ & 0 & 101 & $<0.001$ \\
\hline After C\&D & 24 & $0(0-2.8)$ & 0 & 18 & 0.008 & 40 & $6.5(2.3-20.5)$ & 0 & 101 & \\
\hline After C\&D + UV-C & 24 & $0(0-0)$ & 0 & 1 & & 40 & $0(0-0)$ & 0 & 5 & $<0.001$ \\
\hline
\end{tabular}

No. number, CFU Colony Forming Unit, IQR interquartile range, Min minimum, Max maximum, C\&D cleaning and disinfection, UV-C ultraviolet C 
Table 3 Colony counts per TSA plate containing C. auris in a stationary phase $\left(10^{3} \mathrm{CFUs} / \mathrm{ml}\right)$ with and without UV-C exposure

\begin{tabular}{lllll}
\hline & C. auris & C. auris & C. auris & C. auris \\
& NCPF & NCPF & NCPF & DSM \\
& 8971 & 8977 & 8984 & 21092 \\
$\begin{array}{l}\text { Control (without } \\
\text { UV-C exposure) }\end{array}$ & $>100$ & $>100$ & $>100$ & $>100$ \\
\hline
\end{tabular}

\begin{tabular}{lrrrr}
\hline Day1 (after UV-C) & & & & \\
1 & $>50$ & 35 & 1 & $>50$ \\
2 & $>50$ & $>50$ & 28 & 35 \\
3 & $>50$ & $>50$ & 1 & $>50$ \\
Day2 (after UV-C) & & & & \\
1 & $>50$ & $>100$ & $>50$ & 20 \\
2 & $>50$ & $>100$ & 11 & $>50$ \\
3 & $>100$ & $>100$ & 10 & $>50$ \\
Day3 (after UV-C) & & & & \\
1 & $>50$ & $>50$ & 11 & $>50$ \\
2 & $>50$ & $>50$ & 0 & $>50$ \\
3 & $>50$ & $>50$ & $>50$ & 30
\end{tabular}

Corresponding UV-C doses received during each disinfection cycle ranged from 75 to $100 \mathrm{~mJ} / \mathrm{cm}^{2}$

ensure the total absence of health personnel returning to their nightshift rooms nearby or other individuals who moved in and out the closed area.

In terms of its user-friendliness and simplicity of operation, the device required-in addition to the pre-programming of the area's maps-further preceding steps to start the disinfection process. The user had to select several items in two different apps on the device's control panel, which was not self-explanatory.

\section{Discussion}

The contaminated hospital environment is a reservoir for various pathogens and may thus serve as a source of HAIs [30]. Conventional manual cleaning and disinfection processes are not always sufficient to eliminate the risk posed by contaminated surfaces [3, 8, 9]. Human factors are likely to be a major contributor. Furthermore, during the COVID-19 pandemic, effective standard disinfectants were unavailable in times of crisis [10], indicating the need of new disinfectants or disinfection methods. Most recently, autonomously moving UV-C disinfection devices-UV-C robots-have been developed to overcome these shortcomings.

The present study shows that UV-C irradiation emitted by the robot significantly decreased the residual surface contamination in the waiting areas of two outpatient clinics of a tertiary hospital compared to manual cleaning and disinfection alone. This is in accordance with other studies that have found a significant decrease of the pathogen bioburden in clinical settings by using a robotic UV-C irradiation device $[12,13,15,31]$. Anderson et al. found that the application of UV-C light significantly reduced the presence of Vancomycin-resistant enterococci (VRE) and Clostridium difficile in patient rooms previously occupied by colonized patients compared to baseline (without prior manual cleaning and disinfection) [20]. Similarly, Yang et al. observed a significant reduction of the number of bacteria colonies sampled from different surfaces after UV-C exposure in uncleaned rooms previously occupied by VRE and MRSA carriers [32]. In the present study-despite the fact that not all surfaces achieved full UV-C light exposure-almost all microorganisms still present after manual cleaning and disinfection were eliminated. However the microbial burden on surfaces was low to average on almost all surfaces after

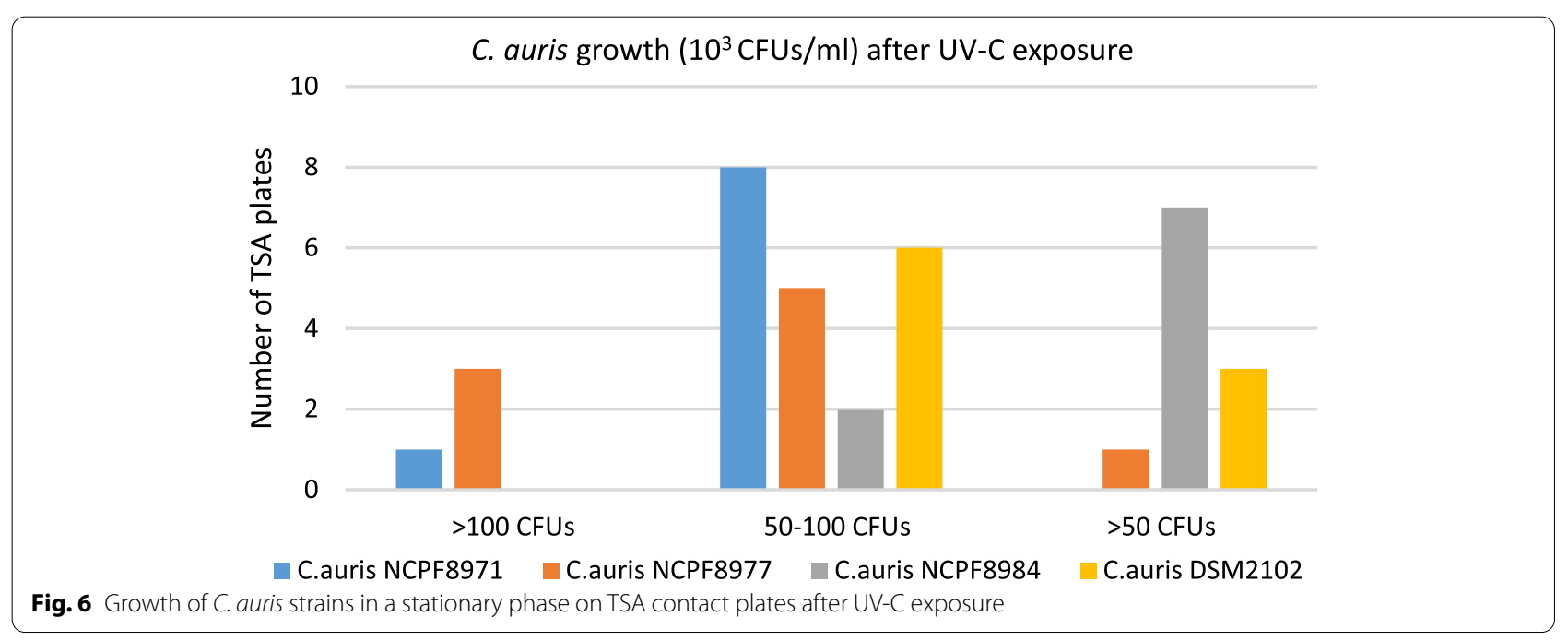


manual cleaning and/or disinfection alone. Especially in the ENT outpatient waiting area, manual cleaning and disinfection had been carried out meticulously, resulting in the recovery of only few CFUs. Insufficient UV-C light exposure might have yielded less effective results in other settings, e.g. with higher surface contamination levels.

Using $C$. auris as a surrogate for microorganisms resilient in the environment, the UV-C disinfection robot tested showed varying effectiveness: For $C$. auris that had been incubated for $24 \mathrm{~h}$ before exposure to UV-C irradiation, growth inhibition was not effectively achieved compared to $C$. auris without prior incubation (for fully exposed areas). However, this may be due to our different sample preparations, mimicking $C$. auris in a lag versus a stationary growth phase. Furthermore, the formation of shadows from the rim of SDA plates drastically decreased the effectiveness of UV-C light. De Groot et al. investigated the effect of different distances (two or four meters) from the UV-C emitting device as well as different cycle times $(5,10,20$ or $30 \mathrm{~min})$ on $C$. auris in vitro, demonstrating strain-dependent effectiveness. Longer $\mathrm{UV}-\mathrm{C}$ exposure and less distance improved the effects of UV-C irradiation [21]. The present study evaluated the effects of UV-C irradiation on $C$. auris delivered by a robot in a clinical setting. These results confirm that longer UV-C exposure is necessary to eradicate $C$. auris, especially when inoculums are high and pathogens have already had time to mature. Thus, it is pivotal to validate the effectiveness of UV-C robotic devices separately for each clinical setting. To achieve this, the robot's settings (distance and duration of exposure) must be carefully calibrated and adapted further until microbiological outcomes are satisfactory. This requires the expertise of a clinical microbiologist and IPC specialist. UV-C sensitive, color-changing indicators measuring the amount of $\mathrm{UV}-\mathrm{C}$ irradiation received must be used as quality controls. Particularly for surfaces or objects that create shadows and cannot be reached by UV-C irradiation, manual cleaning and disinfection are still needed. In the future, design of hospital areas will have to avoid structures creating shadows if UV-C disinfection is applied. Nevertheless, for surfaces beyond the reach of the cleaners, UV-C robots may be useful. Then, to achieve effective UV-C disinfection the UV-C source must be able to move into three dimensions (tilt, move up and down).

The usability of the UV-C robot in the clinical setting was not as satisfactory as expected from a robotic device. This study aimed to evaluate the UV-C robot in action, particularly its usability when integrated into the standard cleaning and disinfection process. UV-C robots are usually advertised as being simple to use with no additional decontamination needed. However, in our own experience, we repeatedly needed technical assistance to operate the robot. Future technological advances are supposed to overcome these failings. In addition to a somewhat complicated programming of the parameter settings, the robot tested was mostly not autonomous and not able to carry out the room disinfection independently. Apart from technical reasons, this dysfunction was also due to the fact that furniture and other objects had been slightly moved between the initial programming and the robot's use. Moreover, despite sealing the areas off during the UV-C disinfection cycles, people moved in and out. Yet, this is normal in the hospital reality. Advances in IT, particularly using artificial intelligence together with high-tech cameras may be the clue for solving these problems. Additionally, further technical developments such as making the UV-C light source more flexible and the robot itself smaller will be necessary in addition to adjustments in the clinical environment to minimize the formation of shadows, thus enabling the proper use of this novel technology.

The decision to use a UV-C disinfection robotic device in clinical settings must be made on the basis of the intended application area, the practicability of use and the additional expected benefit. At the present stage of UV-C robot technology, these robots will be preferably used in areas with stationary furniture that can easily be sealed off to avoid people walking in and out during an ongoing disinfection cycle. Practicability means that the UV-C robot will be operated by trained cleaning staff rather than by an engineer or a technician. An additional expected benefit, however, might be achieved in areas with vulnerable patients or in over-busy areas with highly contaminated surfaces including the risk of multidrugresistant microorganisms, e.g. emergency departments.

There are some limitations to this study. First, showing a reduction in the microbial burden on surfaces is a surrogate outcome. The study design did not allow for an evaluation of the effect of UV-C irradiation in addition to manual cleaning and disinfection on HAI rates compared to manual cleaning and disinfection alone. Next, the classification of the sampling sites into surfaces with a low, average and high microbial burden was made according to our in-house standard, which is used to audit the cleaning efficacy. It refers to the colony count but is not directly associated with patients' outcomes. Further, the cleaning personnel was not blinded to the intervention, which might have affected their behavior, resulting in more thorough cleaning and not reflecting the quality of cleaning in daily practice. Therefore, our results might underestimate the benefit provided by adding a UV-C component. Next, to determine the effects of the UV-C robotic device on $C$. auris, artificially inoculated plates were used as a surrogate for surface-bound contamination. This might not accurately reflect growth patterns 
of $C$. auris on real-world surfaces. Moreover, densities occurring on contaminated hospital surfaces may not be as high, resulting in an underestimation of the ability of UV-C light to kill C. auris.

\section{Conclusion}

The UV-C disinfection model robot tested in our study was not yet ready for everyday use in hospitals due to several technical shortcomings and difficulty of use as well as likely significant additional expense. We also observed persistence of $C$. auris in a stationary phase, indicating that a standard disinfection cycle might not suffice to inactivate more UV-C resistant pathogens, especially when inoculums are high. While UV-C technologies improve surface decontamination results, they do not simplify current processes and can presently only serve as add-on components to manual cleaning and disinfection carried out by trained and audited cleaning staff. However, there is huge potential in this technology once it is further developed.

\begin{abstract}
Abbreviations
C. auris: Candida auris; C\&D: Cleaning and disinfection; CFU: Colony-Forming Unit; ENT: Ear, nose and throat; IPC: Infection prevention and control; HAl: Healthcare-associated infection; MRSA: Methicillin-resistant Staphylococcus aureus; SDA: Sabouraud Dextrose Agar; SOP: Standard operating procedure; STC\&D: Standard terminal room cleaning and disinfection; TSA: Tryptic Soy Agar; UV-C: Ultraviolet-C; VRE: Vancomycin-resistant Enterococcus.
\end{abstract}

\section{Supplementary Information}

The online version contains supplementary material available at https://doi. org/10.1186/s13756-021-00945-4.

Additional file 1. The use of a UV-C disinfection robot in the routine clearning process: a field study in an Academic Hospital. Table S1. Reductions in Colony-Forming Units in the ENT outpatient area after routine cleaning and/or disinfection, and after the use of the UV-C robot. Table S2. Reductions in Colony-Forming Units in the oncology outpatient area after routine cleaning and/or disinfection, and after the use of the UV-C robot. Table S3. ENT outpatient area: Environmental microbiome identified during the study period. Table S4. Oncology outpatient area: Environmental microbiome identified during the study period.

\section{Acknowledgements}

We would like to thank Clean Room Solutions for providing their Ultra Violet Disinfection Robot ${ }^{\circledR}$ (UVD-R) as well as technical support during the study period. Clean Room Solutions did not provide any funding for the study and did not contribute to the study design or writing of the manuscript. We would also like to thank Thi Lan Vi Tran for assisting with the statistical analysis as well as the ESCMID Study Group for Nosocomial Infections (ESGNI) for their valuable input

\section{Authors' contributions}

DM and PE designed the study. ZB prepared the Candida auris samples and summarized the results regarding Candida auris growth inhibition as a result of UV-C exposure. NM and EJ assisted in the execution of the study. FA executed the study, analyzed and interpreted data regarding the effect of UV-C irradiation on environmental contamination and Candida auris growth and wrote the manuscript. DM, PE, EJ and NM revised the manuscript. All authors read and approved the final manuscript.

\section{Funding}

This research did not receive any specific grant from funding agencies in the public, commercial, or not-for-profit sectors.

\section{Availability of data and materials}

The datasets used and/or analyzed during the current study are available from the corresponding author on reasonable request.

\section{Declarations}

Ethics approval and consent to participate

Not applicable.

\section{Consent for publication}

Not applicable.

\section{Competing interests}

The authors certify that they have no affiliations with or involvement in any organization or entity with any financial interest or non-financial interest (such as personal or professional relationships, affiliations, knowledge or beliefs) in the subject matter or materials discussed in this manuscript.

Received: 2 December 2020 Accepted: 26 February 2021

Published online: 29 May 2021

\section{References}

1. Magill SS, Edwards JR, Bamberg W, Beldavs ZG, Dumyati G, Kainer MA, et al. Multistate point-prevalence survey of health care-associated infections. N Engl J Med. 2014;370(13):1198-208.

2. WHO. Report on the endemic burden of healthcare-associated infection worldwide. 2011. https://www.who.int/infection-prevention/publicatio ns/burden_hcai/en/. 05 Nov 2020

3. Dancer SJ. Importance of the environment in meticillin-resistant Staphylococcus aureus acquisition: the case for hospital cleaning. Lancet Infect Dis. 2008;8(2):101-13.

4. Guerrero DM, Nerandzic MM, Jury LA, Jinno S, Chang S, Donskey CJ. Acquisition of spores on gloved hands after contact with the skin of patients with Clostridium difficile infection and with environmental surfaces in their rooms. Am J Infect Control. 2012;40(6):556-8.

5. Hayden MK, Blom DW, Lyle EA, Moore CG, Weinstein RA. Risk of hand or glove contamination after contact with patients colonized with vancomycin-resistant enterococcus or the colonized patients' environment. Infect Control Hosp Epidemiol. 2008;29(2):149-54.

6. Kramer A, Schwebke I, Kampf G. How long do nosocomial pathogens persist on inanimate surfaces? A systematic review. BMC Infect Dis. 2006:6:130.

7. Weber DJ, Anderson D, Rutala WA. The role of the surface environment in healthcare-associated infections. Curr Opin Infect Dis. 2013;26(4):338-44.

8. Carling PC. Evaluating the thoroughness of environmental cleaning in hospitals. J Hosp Infect. 2008;68(3):273-4.

9. Carling PC, Parry MM, Rupp ME, Po JL, Dick B, Von Beheren S. Improving cleaning of the environment surrounding patients in 36 acute care hospitals. Infect Control Hosp Epidemiol. 2008;29(11):1035-41.

10. Kampf G, Scheithauer S, Lemmen S, Saliou P, Suchomel M. COVID19-associated shortage of alcohol-based hand rubs, face masks, medical gloves and gowns - proposal for a risk-adapted approach to ensure patient and healthcare worker safety. J Hosp Infect. 2020;105(3):424-7.

11. Misovic M, Milenkovic D, Martinovic T, Ciric D, Bumbasirevic V, Kravic-Stevovic T. Short-term exposure to UV-A, UV-B, and UV-C irradiation induces alteration in cytoskeleton and autophagy in human keratinocytes. Ultrastruct Pathol. 2013;37(4):241-8.

12. Liscynesky C, Hines LP, Smyer J, Hanrahan M, Orellana RC, Mangino JE. The effect of ultraviolet light on Clostridium difficile spore recovery versus bleach alone. Infect Control Hosp Epidemiol. 2017;38(9):1116-7. 
13. Wong T, Woznow T, Petrie M, Murzello E, Muniak A, Kadora A, et al. Postdischarge decontamination of MRSA, VRE, and Clostridium difficile isolation rooms using 2 commercially available automated ultraviolet-Cemitting devices. Am J Infect Control. 2016;44(4):416-20.

14. Hosein I, Madeloso R, Nagaratnam W, Villamaria F, Stock E, Jinadatha C. Evaluation of a pulsed xenon ultraviolet light device for isolation room disinfection in a United Kingdom hospital. Am J Infect Control. 2016;44(9):e157-61.

15. El Haddad L, Ghantoji SS, Stibich M, Fleming JB, Segal C, Ware KM, et al. Evaluation of a pulsed xenon ultraviolet disinfection system to decrease bacterial contamination in operating rooms. BMC Infect Dis. 2017;17(1):672.

16. Anderson DJ, Chen LF, Weber DJ, Moehring RW, Lewis SS, Triplett PF, et al. Enhanced terminal room disinfection and acquisition and infection caused by multidrug-resistant organisms and Clostridium difficile (the Benefits of Enhanced Terminal Room Disinfection study): a clusterrandomised, multicentre, crossover study. Lancet (London, England). 2017;389(10071):805-14.

17. Health Quality Ontario. Portable ultraviolet light surface-disinfecting devices for prevention of hospital-acquired infections: a health technology assessment. Ont Health Technol Assess Ser. 2018;18(1):1-73.

18. Nerandzic MM, Cadnum JL, Pultz MJ, Donskey CJ. Evaluation of an automated ultraviolet radiation device for decontamination of Clostridium difficile and other healthcare-associated pathogens in hospital rooms. BMC Infect Dis. 2010;10:197.

19. Lindsley WG, MCClelland TL, Neu DT, Martin SB Jr, Mead KR, Thewlis RE, et al. Ambulance disinfection using Ultraviolet Germicidal Irradiation (UVGI): effects of fixture location and surface reflectivity. J Occup Environ Hyg. 2018;15(1):1-12.

20. Anderson DJ, Gergen MF, Smathers E, Sexton DJ, Chen LF, Weber DJ, et al. Decontamination of targeted pathogens from patient rooms using an automated ultraviolet-C-emitting device. Infect Control Hosp Epidemiol. 2013:34(5):466-71.

21. de Groot T, Chowdhary A, Meis JF, Voss A. Killing of Candida auris by UV-C: importance of exposure time and distance. Mycoses. 2019;62(5):408-12.

22. Alfouzan W, Dhar R, Albarrag A, Al-Abdely H. The emerging pathogen Candida auris: a focus on the Middle-Eastern countries. J Infect Public Health. 2019;12(4):451-9.
23. Kordalewska M, Perlin DS. Identification of drug resistant Candida auris. Front Microbiol. 2019;10:1918.

24. Calvo B, Melo AS, Perozo-Mena A, Hernandez M, Francisco EC, Hagen F, et al. First report of Candida auris in America: clinical and microbiological aspects of 18 episodes of candidemia. J Infect. 2016;73(4):369-74.

25. Schelenz S, Hagen F, Rhodes $J$, Abdolrasouli A, Chowdhary A, Hall A, et al. First hospital outbreak of the globally emerging Candida auris in a European hospital. Antimicrob Resist Infect Control. 2016;5:35.

26. Cadnum JL, Shaikh AA, Piedrahita CT, Jencson AL, Larkin EL, Ghannoum MA, et al. Relative resistance of the emerging fungal pathogen Candida auris and other Candida species to killing by ultraviolet light. Infect Control Hosp Epidemiol. 2018;39(1):94-6.

27. Ponnachan P, Vinod V, Pullanhi U, Varma P, Singh S, Biswas R, et al. Antifungal activity of octenidine dihydrochloride and ultraviolet-C light against multidrug-resistant Candida auris. J Hosp Infect. 2019;102(1):120-4.

28. O'Meara S. Mechanical medics to the rescue. Nature. 2020;582:49.

29. Adams E, Quinn M, Tsay S, Poirot E, Chaturvedi S, Southwick K, et al. Candida auris in healthcare facilities, New York, USA, 2013-2017. Emerg Infect Dis J. 2018;24(10):1816.

30. Suleyman G, Alangaden G, Bardossy AC. The role of environmental contamination in the transmission of nosocomial pathogens and healthcareassociated infections. Curr Infect Dis Rep. 2018;20(6):12.

31. Doll M, Morgan DJ, Anderson D, Bearman G. Touchless technologies for decontamination in the hospital: a review of hydrogen peroxide and UV devices. Curr Infect Dis Rep. 2015;17(9):498.

32. Yang J-H, Wu U-I, Tai H-M, Sheng W-H. Effectiveness of an ultraviolet-C disinfection system for reduction of healthcare-associated pathogens. J Microbiol Immunol Infect. 2019;52(3):487-93.

\section{Publisher's Note}

Springer Nature remains neutral with regard to jurisdictional claims in published maps and institutional affiliations.
Ready to submit your research? Choose BMC and benefit from:

- fast, convenient online submission

- thorough peer review by experienced researchers in your field

- rapid publication on acceptance

- support for research data, including large and complex data types

- gold Open Access which fosters wider collaboration and increased citations

- maximum visibility for your research: over 100M website views per year

At BMC, research is always in progress.

Learn more biomedcentral.com/submissions 